\title{
Source, Triggers and Clinical Implications of Hyperlactemia in Patients Undergoing Mitral Valve Surgery Using Custodiol Cardioplegia
}

\author{
Raimondo Ascione ${ }^{1}$, Andrea Venturini ${ }^{2}$, Elvio Polesel ${ }^{2}$, Domenico Mangino ${ }^{2}$, \\ Chiara Zanchettin ${ }^{2}$, Giampaolo Zoffoli ${ }^{2}$, Gianni Angelini ${ }^{1}$, M.-Saadeh Suleiman ${ }^{1}$ \\ ${ }^{1}$ CSIRO Bristol Heart Institute, University of Bristol, Bristol, UK \\ ${ }^{2}$ U.O. di Cardiochirurgia, Ospedale Civile "Umberto I", Venice, Italy \\ Email: r.ascione@bristol.ac.uk
}

Received June 6, 2013; revised July 4, 2013; accepted July 18, 2013

Copyright (C) 2013 Raimondo Ascione et al. This is an open access article distributed under the Creative Commons Attribution License, which permits unrestricted use, distribution, and reproduction in any medium, provided the original work is properly cited.

\begin{abstract}
Background: Postoperative blood hyperlactaemia is an indicator of organ anaerobic metabolism and is associated with morbidity after cardiac surgery. This prospective study aims to explore the source, triggers and clinical implications for hyperlactaemia in patients undergoing mitral valve surgery using Custodiol cardioplegia. Methods: Twenty consecutive elective patients undergoing open-heart surgery for mitral valve repair/replacement using Custodiol (based on Bretschneider's HTK-solution) cardioplegia were recruited. A serial measurement of arterial blood lactate was performed. Pre-, intra- and post-operative clinical data were obtained and cardiac injury was determined by serial plasma measurement of postoperative release of CK-MB. Results: There were no in-hospital deaths. Most of the patients $(n=16)$ needed intraoperative direct current cardioversion to treat ventricular arrhythmias or post-operative vasopressors $(n=13)$ to treat vasoplegia. There was significant cardiac injury as determined by the marked increase of serum CK-MB $(\mathrm{p}<0.05)$. A significant $(\mathrm{p}<0.05)$ increase in blood lactate was found to follow a biphasic profile. The first peak (from $0.54 \pm$ 0.03 to $1.3 \pm 0.07 \mathrm{mM}$ ) was observed immediately following the release of the aortic cross-clamp and remained high for 1 hour. This was followed by a second peak at 12 hours postoperatively $(1.9 \pm 0.2 \mathrm{mM})$. The second rise in lactate was seen only in patients that required post-operative vasopressors $(1.3 \pm 0.2 \mathrm{vs} 2.2 \pm 0.3 \mathrm{mM}, \mathrm{p}<0.05)$, in whom a significant late decrease in CVP was also observed (12.2 \pm 1.0 to $7.7 \pm 1.0$ for 1 and 12 hours postoperative, respectively). Hyperlactaemia did not correlate with any other variables including CK-MB levels, cross-clamp or cardiopulmonary bypass time. Conclusions: In patients undergoing mitral valve surgery with Custodiol cardioplegia there is marked cardiac injury and a biphasic release of blood lactate. The initial peak in lactate occurs immediately following unclamping the aorta and is likely to be of organ (e.g. heart and lungs) origin. A second peak is only seen in patients requiring postoperative vasopressors to treat vasoplegia. Hyperlactaemia following mitral valve surgery with Custodiol cardioplegia does not seem to be related to myocardial injury as expressed by CK-MB release.
\end{abstract}

Keywords: Mitral Valve; Blood Lactate; Cardiac Injury; Custodiol

\section{Introduction}

Cardioplegic arrest (CA) and cardiopulmonary bypass (CPB) during cardiac surgery are associated with ischemic/hypoxic stress seen in cardiac and non-cardiac tissue. Cross-clamping the aorta and infusion of cardioplegic solutions render the heart (and the lungs) ischemic [1-3]. The addition of large volume of pump prime to the patient's circulation at the beginning of CPB decreases arterial oxygen content causing the release vasoconstrictors and therefore results in reduced oxygen delivery to peripheral (e.g. skeletal muscle) and visceral (e.g. GI tract) tissues [4-6]. In skeletal muscle the effect of CPB involves changes in metabolic activity as well as changes in blood flow $[7,8]$. A consequence of ischemic/hypoxic stress (anaerobic metabolism) associated with $\mathrm{CA}$ and CPB is the accumulation of lactate in cardiac $[9,10]$ as well as in peripheral (skeletal muscle) and visceral tissue [11]. Significant proportion of the lactate accumulated in tissue effluxes using the lactate transporter [12]. Therefore measurement of blood lactate levels is used to determine the extent of anaerobic metabolism and indirectly infer the extent of tissue oxygenation/perfusion. 
However, the interpretation of elevated blood lactate levels following cardiac surgery is complex as the levels are altered by amongst other factors inter-organ substrate flux, hepatic lactate uptake, hypovolemia, low cardiac output (LCO), peripheral arterial thrombosis) [13]. Evidence suggests that elevated blood lactate levels (hyperlactaemia) observed following cardiac surgery may correlate with the duration of CPB [14], it is relatively frequent and is associated with an increased postoperative morbidity $[15,16]$. There is little knowledge about changes in blood lactate during and after mitral valve surgery using Custodiol (Bretschneider's histidine-tryptophanketoglutarate) cardioplegia. This cardioplegia is supposed to provide good buffering capacity and enhances anaerobic energy production during ischaemic cardioplegic arrest $[17,18]$. Cardioplegic solutions are likely to have different systemic effects due to their composition and the difference in their volume. In this prospective pilot study we aim to explore the potential source, triggers and clinical implications for hyperlactaemia in patients with mitral valve disease undergoing mitral valve surgery using Custodiol cardioplegia.

\section{Materials and Methods}

A consecutive series of adult patients with mitral valve disease undergoing elective mitral valve repair/replacement were prospectively recruited. The study was approved by the Hospital Ethics Committee, and all patients gave informed consent.

\subsection{Surgical and Anesthetic Techniques}

A standard CPB circuit was used with a $40-\mu \mathrm{m}$ filter, a Stockert roller pump and a fibre membrane oxygenator (Dideco, Sorin, Cobe). Priming solution included 1000 $\mathrm{mL}$ of balanced electrolyte solution $(\mathrm{pH} 7.4), 500 \mathrm{~mL}$ plasma expanding reagent (6\% hydroxyethyl starch), 100 $\mathrm{mL}$ 18\% mannitol, and 5000 IU Heparin. Non-pulsatile flow was used at a rate of $2.4 \mathrm{l} / \mathrm{m}^{2} / \mathrm{min}$. Systemic tem0 perature was kept at $34^{\circ} \mathrm{C}$. Heparin IV was used at a dose of $300 \mathrm{UI} / \mathrm{Kg}$ to achieve a target ACT of $480 \mathrm{sec}$ or more. For induction and maintenance of anesthesia, Propofol infusion was used at $2-2.8 \mu \mathrm{g} / \mathrm{ml}(5.4-7.5 \mathrm{mg} / \mathrm{Kg} / \mathrm{min})$ combined with Remifentanyl infusion at $3.5-7.60 \mu \mathrm{g} / \mathrm{ml}$ $(0.13-0.28 \mu \mathrm{g} / \mathrm{Kg} / \mathrm{min})$ using a Diprifusor pump. Neuromuscular blockade was achieved with $0.6 \mathrm{mg} / \mathrm{Kg}$ of Rocuronium infused at $0.12 \mathrm{mg} / \mathrm{Kg} / \mathrm{min}$. Normocapnia was maintained and the lungs were ventilated with a mixture of air $/ \mathrm{O}_{2}=0.5$, with low flow ventilation technique. Target mean arterial blood pressure was $65-70$ mmHg. On removal of the aortic cross clamp the need for direct current (DC) cardioversion was dictated by the occurrence of malignant ventricular arrhythmias. Fol- lowing transfer to intensive care unit sedation was maintained with Propofol infusion at $1-2 \mu \mathrm{g} / \mathrm{ml}$ combined with Remifentanyl infusion at $2.0-2.5 \mu \mathrm{g} / \mathrm{ml}$. Weaning from mechanical ventilator was according to a predefined protocol. Adequate postoperative systemic perfusion was achieved by aiming for a mean blood pressure of 65 $\mathrm{mmHg}$ or more. Volemia was maintained with fluid resuscitation targeting a central venous pressure (CVP) of $12-15 \mathrm{mmHg}$ for ventilated patients and $8-12 \mathrm{mmHg}$ for non-ventilated patients. The occurrence of hypotension (without sepsis) despite adequate fluid resuscitation, together with evidence of perfusion abnormalities (i.e. oliguria) was regarded as vasoplegia. This was treated with the vasopressor of choice Noradrenaline (dosages range $0.02-1.5 \mathrm{mcg} / \mathrm{kg} / \mathrm{min}$ ). Occurrence of low cardiac output (LCO) was treated with Dopamine (dosage range $5-20 \mathrm{mcg} / \mathrm{kg} / \mathrm{min}$ ) or Enoximone (4 $\mu \mathrm{g} / \mathrm{kg} / \mathrm{min})$.

\subsection{Cardioplegic Techniques}

A single shot ice-cold Custodiol cardioplegia was delivered directly in the aortic root for approximately 10 min at a rate of $200 \mathrm{ml} / \mathrm{min}$ giving a mean total infusion volume of $2000 \mathrm{ml}$.

\subsection{Blood Lactate}

This was assessed serially by measuring plasma lactate levels at baseline (pre-op), intraoperatively (at aortic cross clamp release and at 30 minutes of reperfusion), and postoperatively (at 1, 6, 12, 24 and 48 hours).

\subsection{Myocardial Injury}

Serial release of Creatine Kinase (CK-MB) was used as a marker of myocardial injury. For this purpose blood samples were collected from the radial artery line at 1, 6, 12, 24 and 48 hours postoperatively.

\subsection{Generic In-Hospital Outcome}

A full set of in-hospital outcomes were monitored. These also included generic postoperative complications such as myocardial infarction, low cardiac output syndrome, need for vasopressors and/or inotropic support, arrhythmia, ST changes, renal, respiratory, and neurological complications, total fluid balance, total blood loss, transfusion requirement, ventilation time, length of intensive care and hospital stay.

\subsection{Management of Pre-Operative Medications}

Preoperative medications including $\alpha$-blockers, diuretics, anti-hypertensives, and calcium channel blockers were routinely omitted on the day of surgery. ACE-inhibitors were withdrawn on the evening before the operation. 


\subsection{Statistical Analysis}

Data were expressed as mean \pm standard error (SE). Comparison between continuous variables for paired groups was made using a non-parametric test (Wilcoxon's signed rank test). Comparison between nominal variables was made using Chi Square test and significance determined using Fisher's exact test. Correlation coefficient was calculated and significance determined by use of Fisher's $r$ to z. All statistical analyses mentioned were performed with the aid of a computerized software package, Statview for Windows (SAS Institute Inc., Cary, NC, USA).

\section{Results}

\subsection{Clinical Outcome}

Preoperative patients' demographics are shown in Table 1. 2.

Key intra- and postoperative data are shown in Table

There were neither in-hospital deaths nor major neurological, respiratory, renal, and gastrointestinal compli-

Table 1. Preoperative data.

\begin{tabular}{|c|c|}
\hline Variable & Total number $(\mathrm{n}=\mathbf{2 0})$ \\
\hline Male/Female & $15 / 5$ \\
\hline Age (years) & $53 \pm 3$ \\
\hline $\operatorname{BSA}\left(\mathrm{m}^{2}\right)$ & $1.89 \pm 0.04$ \\
\hline Hypertension & 9 \\
\hline Ejection fraction (\%) & $64 \pm 1$ \\
\hline \multicolumn{2}{|l|}{ NYHA class } \\
\hline I & 6 \\
\hline II & 13 \\
\hline III & 1 \\
\hline History of AF & 2 \\
\hline History of heart failure & 2 \\
\hline \multicolumn{2}{|l|}{ Degree of MR } \\
\hline $3+/ 4$ & 11 \\
\hline $4+/ 4$ & 9 \\
\hline \multicolumn{2}{|l|}{ Type of MR } \\
\hline Degenerative & 16 \\
\hline Rheumatic & 3 \\
\hline Both & 1 \\
\hline \multicolumn{2}{|l|}{ Associated procedures } \\
\hline Tricuspid valve repair & 6 \\
\hline AF ablation & 1 \\
\hline
\end{tabular}

Data are presented as mean $\pm \mathrm{SE}$ or as number of patients. BSA, body surface area; AF, atrial fibrillation; MV, mitral valve; NYHA, New York Heart Association; CPB, cardiopulmonary bypass; ICU, intensive care unit. cations. No episodes of low cardiac output or myocardial infarction were observed. Sixteen patients $(80 \%)$ required intra-operative DC cardioversion on releasing the aortic cross-clamp due to ventricular arrhythmias. Thirteen patients $(65 \%)$ suffered postoperative vasoplegia according to predefined criteria and required usage of vasopressors.

\subsection{Cardiac Injury}

There was significant cardiac injury as determined by the marked increase of serum CK-MB after surgery (Figure 1(A)).

It has been suggested that CK-MB levels of more than $100 \mathrm{ng} / \mathrm{ml}$ has $95 \%$ specificity in relation to cardiac events whilst $45 \mathrm{ng} / \mathrm{ml}$ has $76 \%$ specificity [19]. Peak CK-MB at $>45 \mathrm{ng} / \mathrm{ml}$ was seen in 19 patients whilst only 2 had levels $>100 \mathrm{ng} / \mathrm{ml}$. These levels were seen on average at 1,6 and 12 hours postoperatively. Release of CK-MB did not correlate with the need for intraoperative DC cardioversion or need for postoperative vasopressors (Figures 1(B) and (C)).

\subsection{Release of Blood Lactate}

A time dependent increase in blood lactate was seen immediately following release of aortic cross clamp and remained elevated at 48 hours postoperatively compared to pre-operative levels (Figure 2). The increase in blood lactate was found to follow a biphasic profile. The first peak was sharp and occurred soon after the release of the aortic cross clamp, remaining high for the following 30 -

Table 2. Intra and post-operative data.

\begin{tabular}{cc}
\hline Variable & $\mathbf{n}=\mathbf{2 0}$ \\
\hline CPB time (min) & $127 \pm 6$ \\
Cross-clamp time (min) & $109 \pm 5$ \\
ICU stay (hours) & $50 \pm 7$ \\
Hospital stay (days) & $12.6 \pm 2.6$ \\
Intra-operative DC cardioversion & 16 \\
Sinus rhythm on weaning from CPB & 18 \\
Inotropic support on weaning from bypass & 7 \\
Postoperative arrhythmia & 8 \\
Total blood loss (ml) & $1051 \pm 145$ \\
ST elevation in ICU & 1 \\
Temporary pacing in ICU & 1 \\
\% Ejection fraction (5 ${ }^{\text {th }}$ day) & 13 \\
Blood loss (ml) & $13.7 \pm 3.3$ \\
Ventilation time (hours) & $63 \pm 2$ \\
\hline Use of vasopressors (noradrenaline) in ICU & $\mathrm{CPB}$ \\
\hline
\end{tabular}

Data are presented as mean $\pm \mathrm{SE}$ or as number of patients. $\mathrm{CPB}$, cardiopulmonary bypass; ICU, intensive care unit; DC, direct current. 
60 minutes. This was followed by a further slow rise in blood lactate that peaked at 12 hours postoperatively. However, even at 48 hours postoperatively, the lactate levels remained significantly higher than preoperative levels.
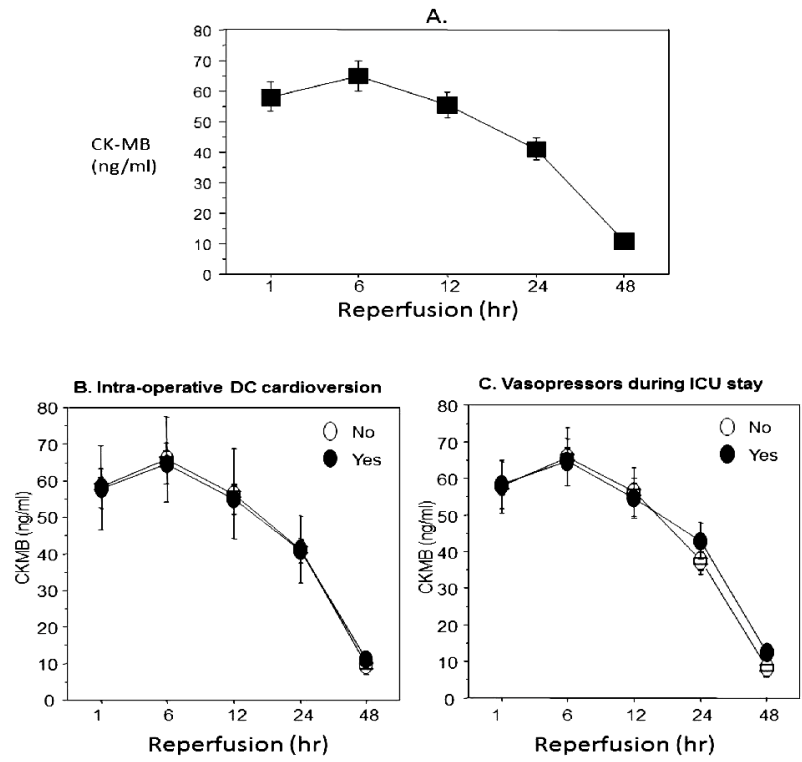

Figure 1. Cardiac injury (postoperative CK-MB release) in patients undergoing mitral valve surgery. Data are presented as mean \pm SE for CK-MB levels for all patients (A) and comparing the effect of DC cardioversion (B) and use of vasopressors during ICU stay (C).

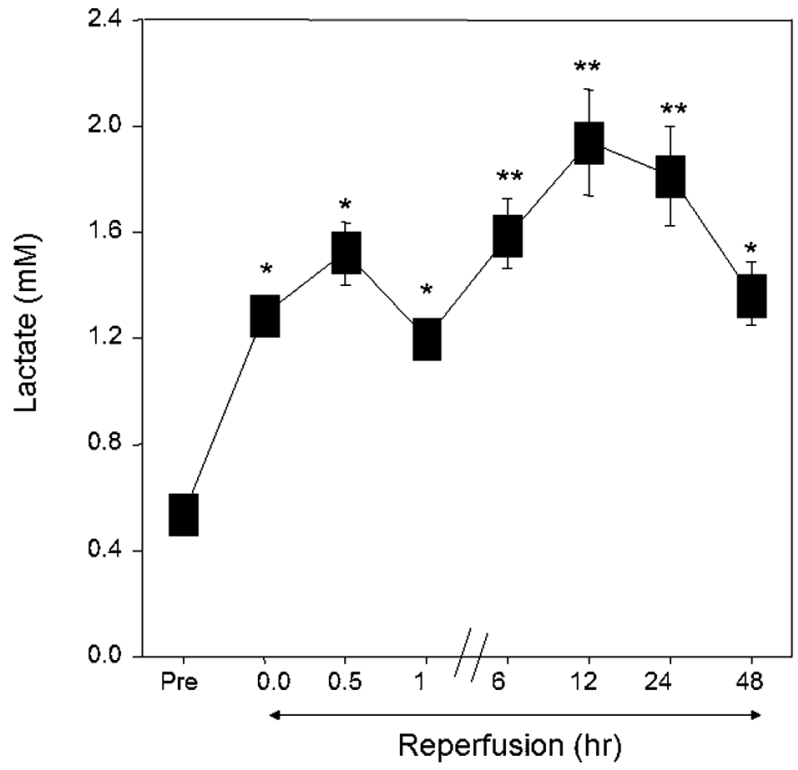

Figure 2. Time-dependent increase in blood lactate during surgery. A significant increase in blood lactate was seen immediately following the release of aortic cross clamp. A second slow rise in blood lactate was seen after 1 hour reperfusion. Data are presented as mean $\pm \mathrm{SE}$. *vs basal blood levels; ** vs basal and 1 hour levels.

\subsection{Early Phase of Hyperlactatemia and Myocardial Ischemic Stress/Injury}

There was no correlation between blood lactate levels and cardioplegic arrest time as shown in Figure 3(A). This was also confirmed when aortic cross-clamp time was correlated with the actual increase in lactate at each time point (data not shown). The finding that cardioplegic arrest time did not correlate with blood lactate levels is consistent with the finding that cardiac injury (peak CK-MB levels) also did not correlate with early elevation in lactate (Figure 3(B)). None of the other preintra- or post-operative clinical measurements including intraoperative DC cardioversion, need for postoperative vasopressors, arrhythmia, need for pacing, blood loss, ventilation time, ICU stay or hospital stay correlated with the early peak of lactate.

\subsection{Delayed Phase of Blood Hyperlactaemia and Post-Operative Vasoplegia}

We found a strong correlation between the late second slow rise in blood lactate and the post-operative use of vasopressors (Figure 4). This relationship was further observed when using lactate value of $1.4 \mathrm{mM}$ as a cut off
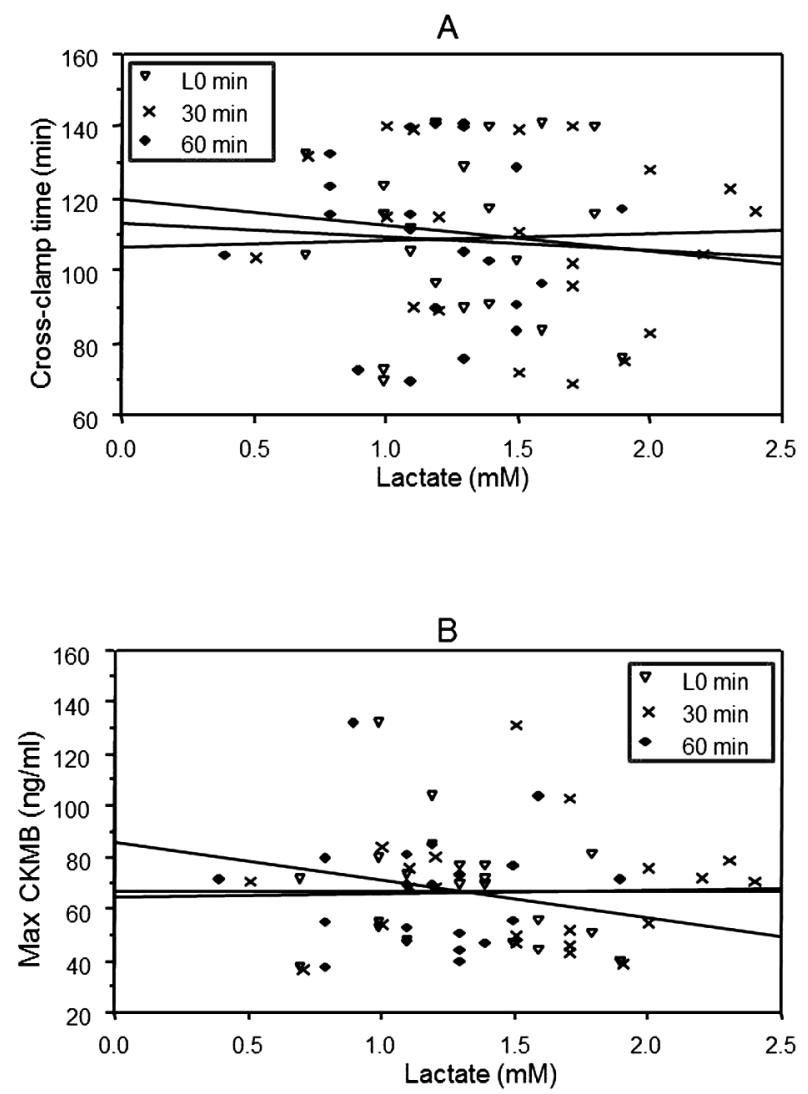

Figure 3. Scattergrams showing the relationship between lactate levels at $0,0.5$ and 1 hour against cross-clamp time (A) and cardiac injury (B). 


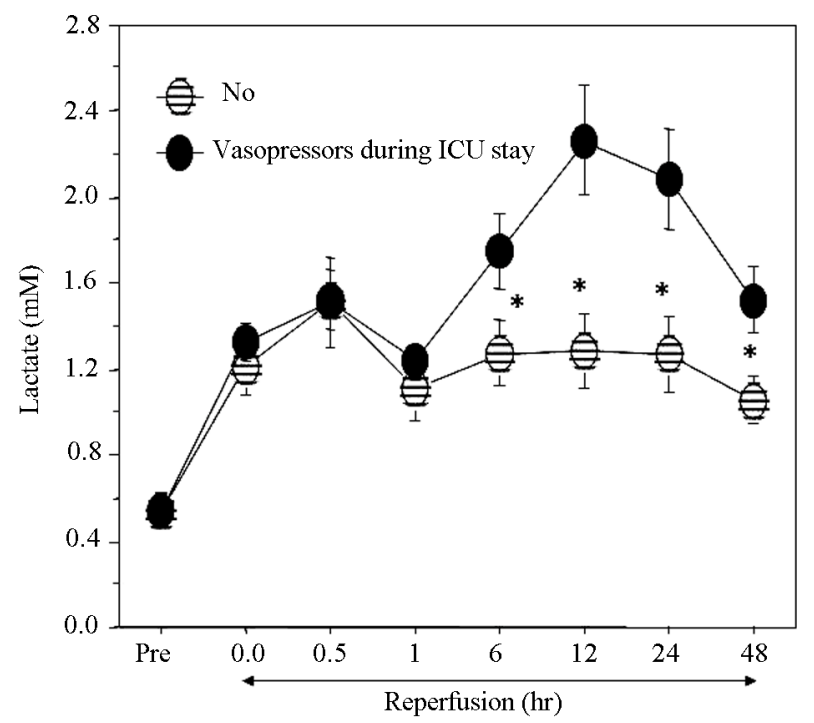

Figure 4. Changes in blood lactate during surgery measured in patients with or without use of vasopressors during ICU stay. Data are presented as mean $\pm \mathrm{SE}$. "vs values for inotropic support at the corresponding time.

(approximately separating the slow and the delayed phase). This approach showed that lactate levels $>1.4$ $\mathrm{mM}$ occurred particularly in patients in whom the need for vasopressors was prolonged (Figure 5(A)).

In addition, in patients with prolonged postoperative need for vasopressor the values of CVP were significantly lower in correspondence of the late phase of hyperlactaemia despite adequate volume replacement (Figure 6). On the contrary, there was no relationship between the late peak of blood lactate and other parameters including cross-clamp and CPB time or CK-MB release (Figure 5(B)). In addition, there was a trend for an association (not significant) between levels of plasma lactate and changes in volemia due to either blood loss or total fluid balance (24 hr) (Figures 7(c) and (d)).

\section{Discussion}

This work reports novel findings highlighting the relationship between levels of blood lactaemia, cardiac injury (as expressed by release of CK-MB) and clinical outcome in elective patients undergoing mitral valve surgery using Custodiol cardioplegia. It suggests that a significant increase in blood lactate is seen immediately upon release of aortic cross-clamp and this level is maintained for up to 48 hours postoperatively. Of note, a late slow increase in blood lactate that peaked at 12 hours after surgery was only observed in patients requiring postoperative vasopressors to treat vasoplegia. However, the increase in blood lactate at all-time points did not correlate with myocardial injury or other measured clinical outcome parameters.

The early phase increase in plasma lactate is likely to

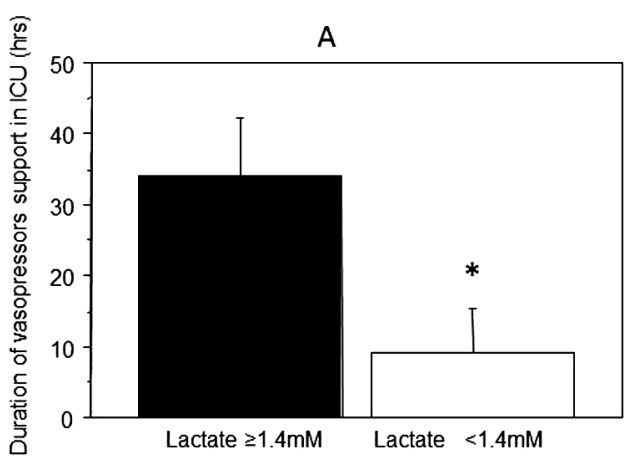

B

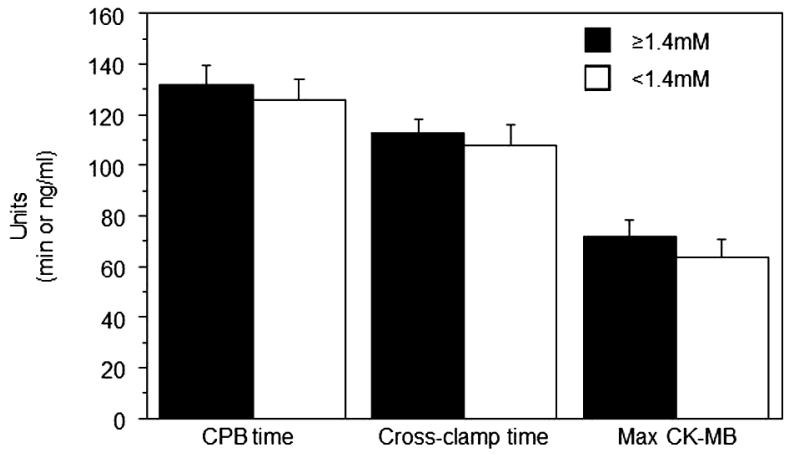

Figure 5. Duration of vasopressor support in ICU (A) or CPB, cross-clamp time and cardiac injury (CK-MB release) (B) divided into patients that had high or low lactate measured at 12 hours postoperatively. Data are presented as mean $\pm \mathrm{SE} .{ }^{*}$ vs high lactate levels.

be due in part to myocardial (and probably pulmonary) lactate that had accumulated during the cardioplegic arrest and CPB. Detrimental effects of CPB and CA are potential sources of lactate release during early phase of hyperlactatemia. During CA, the heart is rendered ischemic where anaerobic metabolism (and lactate production) becomes the main source of energy production. Accumulated lactate is known to efflux the heart $\left(\right.$ with $\mathrm{H}^{+}$) immediately following reperfusion [12]. Work using microdialysis has demonstrated that during cardioplegic arrest and CPB the heart accumulates lactate in the vasculature $[9,10]$. Lactate accumulated in heart cells during ischemia is known to be released immediately following upon reperfusion [12]. Since the heart is highly metabolically active it is likely to be the main organ sustaining ischemic stress during $\mathrm{CPB}$ and cardioplegic arrest. However, since our study suggests that plasma lactate levels are maintained for a further 1 hour, other organs would also contribute likely by suffering ischemia during CPB.

Our study suggests that the use of Custodiol cardioplegia in patients undergoing mitral valve surgery is associated with significant myocardial injury. However, the finding that plasma lactate levels immediately following unclamping the aorta do not correlate with cardiac injury as expressed by $\mathrm{CK}-\mathrm{MB}$ release is potentially surprising. 


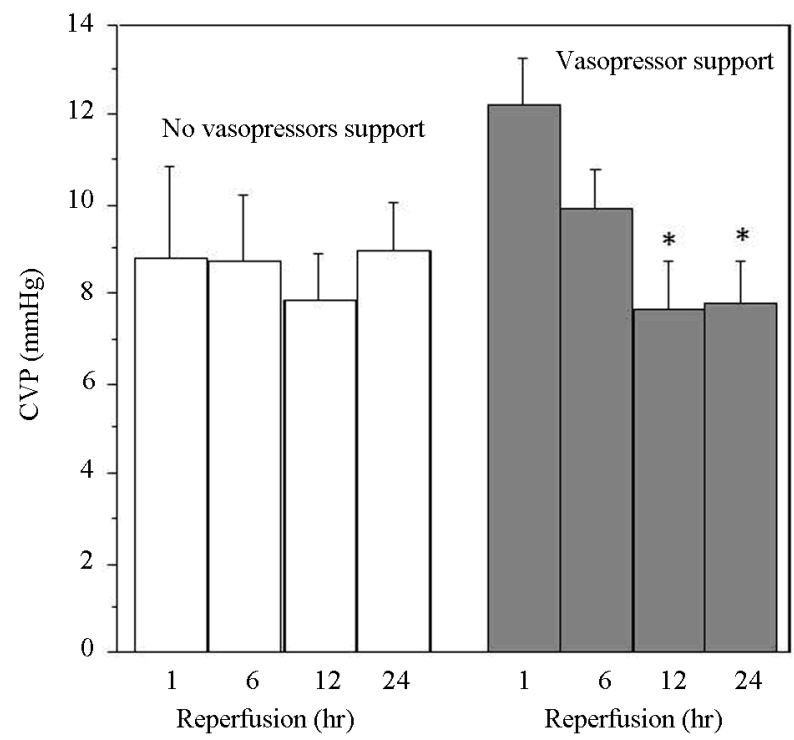

Figure 6. Changes in CVP divided according to requirement of vasopressors in ICU. Data are presented as mean \pm SE. * vs corresponding CVP measured at $1 \mathrm{hr}$.

In our opinion this could be due to the fact that crossclamp time was relatively long as most of the patients (n $=17$ ) had cross-clamp time between $\sim 90-140 \mathrm{~min}$. Alternatively, this could simply reflect the fact that following an acute cardiac ischemic event it takes much longer for the CK-MB levels to rise in the plasma compared to the time required for the plasma lactate. Furthermore, while the levels of lactate are directly linked to ischemia, the release of CK-MK in patients undergoing mitral valve surgery might also reflect the specific need of entering the left atrium via a long incision across its muscular wall (direct iatrogenic injury) to operate on the mitral valve (plus a further incision of the right atrial wall for those undergoing concomitant tricuspid valve repair). This could lead to an extra release of CK-MB over and above that associated with cardioplegic arrest related injury. The latter point might also explain why in our study cardiac injury as expressed by CK-MB plasma levels did not correlate with aortic cross-clamp time (cardiac ischemia). Of note, other perioperative interventions (e.g. intraoperative DC cardioversion) did not alter level of plasma lactate suggesting that the increase is most likely due entirely to the long period of ischemic cardioplegic arrest.

Of interest, this study also show for the first time that the increase in blood lactate in patients undergoing mitral valve surgery with Custodiol cardioplegia follows a biphasic profile (early and sharp first peak followed by a late and slow secondary peak) where the late slow increase was only seen in patients needing postoperative vasopressors, which might have implications for their effective management. We noted that in these patients
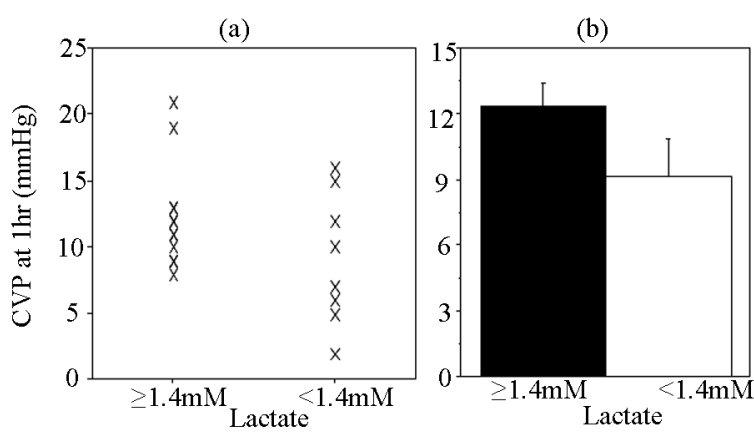

(c)

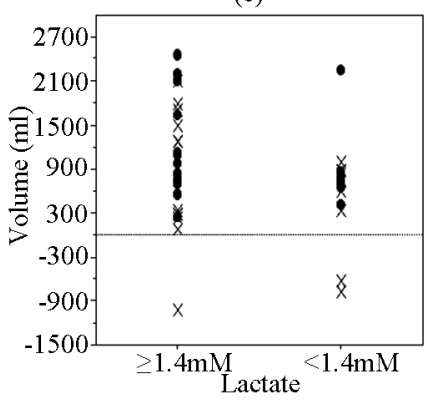

(d)

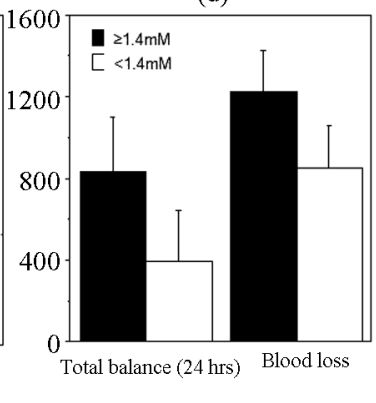

Figure 7. (a) shows the individual values for CVP in individual patients divided into high or low lactate levels whilst; (b) shows the same data presented as mean \pm SE. Blood loss (closed circle) and total fluid balance ( $24 \mathrm{hr}$ ) levels in individual patients divided into high or low lactate levels (c); Same data is shown as mean \pm SE (d).

the late second peak of blood lactate did not correlate with late levels of CK-MB and it reached its highest value at 12 hours postoperatively.

It remains uncertain whether this late slow rise in lactate is due to CPB-induced tissue metabolic stress or due to side effects of vasopressors or both. We feel that our study is too small to answer this important question. However, when looking at the literature, it is evident that the significance, source and clinical implications of changes in blood lactate levels during cardiac surgery remain still unresolved. Elevated levels of blood lactate have been observed also following off-pump coronary artery bypass grafting, although to less extent than the conventional coronary surgery with CPB (CABG) [20]. Others have suggested that hypothermia, particularly in skeletal muscles, could be triggering lactate release in these patients [21]. Postoperative lactate release has been shown by others to be affected by degree and type of vasoconstrictor and/or inotropic support. The use of betablockade peri-operatively in the presence of phenylephrine as vasopressor did not alter arterial lactate up to 16 hours post operatively [22]. Furthermore, in patients undergoing $\mathrm{CABG}$ only the use of noradrenaline but not milrinone triggered increase in blood lactate during surgery [23].

In our practice, we used the vasopressor noradrenaline in accordance with the accepted practice of maintaining 
adequate systemic perfusion (aiming for a mean blood pressure of $65 \mathrm{mmHg}$ or above). The use of noradrenaline was prompted by the occurrence of hypotension (without sepsis) despite adequate fluid resuscitation, together with evidence of perfusion abnormalities (i.e. oliguria). This condition is regarded by many as vasoplegic syndrome [24]. Noradrenaline is known to produce a dose-dependent increase in systemic vascular resistance. Although the drug can stimulate cardiac b-receptors over a wide range, the cardiac output is increased only at low doses. At high dose rates, the cardiac output decreases in response to the vasoconstriction and increased afterload. Hence, the administration of any vasoconstrictor agent carries a risk of hypoperfusion and ischemia involving any tissue bed or vital organ. It might be therefore that the observed late slow late peak in blood lactate in our study is related to excessive systemic vasoconstriction. Naturally, for any condition that requires vasoconstrictor drugs to maintain a blood pressure, it is difficult to distinguish adverse drug effects and adverse disease effects with potentially subsequent little or no room for therapeutic manipulations. Of note, thirteen patients $(65 \%)$ in our study appeared to suffer vasoplegia during the postoperative stay since they required a prolonged use of noradrenaline for more than 5 hours despite normal cardiac output status. One might argue that the use of Custodiol in our study could have triggered this vasoplegiatype condition leading to the observed degree of systemic anaerobic metabolism. Vasoplegic syndrome is a welldescribed form of vasodilatory shock that potentially can occur after separation from CPB [24]. It is a state of low systemic arterial pressure despite high cardiac output and adequate fluid resuscitation characterized by markedly low systemic vascular resistance [25]. The reported incidence ranges from $9 \%$ to $44 \%$, yet the origin has not been completely elucidated [24]. There are already reports of vascular complications associated with the use of Custodiol. These include low efficacy at protecting human and pig liver endothelial cells [26,27] and ability to induce red blood cell deformability possibly by altering cell membrane [28]. In keeping with this evidence, our findings appear to support the notion that Custodiol cardioplegia might be associated with a postoperative vasoplegic syndrome, however larger studies are required to ascertain the risk/benefit balance of using Custodiol cardioplegia in patients undergoing mitral valve surgery.

In conclusion, this study suggests that there might be a significant degree of myocardial injury associated with the use of Custodiol cardioplegia in patients undergoing mitral valve surgery. In addition, there appear to be a biphasic release of blood lactate with an initial peak occurring immediately following unclamping the aorta and likely to be of organ (e.g. heart and lungs) origin. A second peak is only seen in patients requiring postoperative vasopressors to treat a vasoplegic-type of syndrome. Hyperlactaemia following mitral valve surgery with Custodiol cardioplegia does not seem to be related to myocardial injury as expressed by CK-MB release. Larger studies are warranted to confirm these findings.

\section{Acknowledgements}

The NIHR Bristol Biomedical Research Unit in Cardiovascular Disease, Garfield Weston Trust and the British Heart Foundation supported this study. We would like to thank Drs A. Asta, A. Terrini, A. Bossi, and Mrs S. Capuozzo of the U.O. di Cardiochirurgia, Ospedale Civile "Umberto I", Venezia-Mestre, Italy, and all members of staff at both institutions for their valuable help and support in carrying out this study.

\section{REFERENCES}

[1] M.-S. Suleiman, K. Zacharowski and G. D. Angelini, "Inflammatory Response and Cardioprotection during OpenHeart Surgery: The Importance of Anaesthetics," British Journal of Pharmacology, Vol. 153, No. 1, 2008, pp. 2133.

[2] R. Ascione, M.-S. Suleiman and G. D. Angelini, "Retrograde Hot-Shot Cardioplegia in Patients with Left Ventricular Hypertrophy Undergoing Aortic Valve Replacement," The Annals of Thoracic Surgery, Vol. 85, No. 2, 2008, pp. 454-458. doi:10.1016/j.athoracsur.2007.08.039

[3] H. Imura, M. Caputo, K. Lim, M. Ochi, M.-S. Suleiman, K. Shimizu and G. D. Angelini, "Pulmonary Injury after Cardiopulmonary Bypass: Beneficial Effects of Low-Frequency Mechanical Ventilation," The Journal of Thoracic and Cardiovascular Surgery, Vol. 137, No. 6, 2009, pp. 1530-1537.

[4] A. Uusaro, E. Ruokonen and J. Takala, "Splanchnic Oxygen Transport after Cardiac Surgery: Evidence for Inadequate Tissue Perfusion after Stabilization of Hemodynamics," Intensive Care Medicine, Vol. 22, No. 1, 1996, pp. 26-33. doi:10.1007/BF01728327

[5] R. Ascione, S. Talpahewa, C. Rajakaruna, B. C. Reeves, A. T. Lovell, A. Cohen and G. D. Angelini, "Splanchnic Organ Injury during Coronary Surgery with or without Cardiopulmonary Bypass: A Randomized, Controlled Trial," The Annals of Thoracic Surgery, Vol. 81, No. 1, 2006, pp. 97-103. doi:10.1016/j.athoracsur.2005.06.038

[6] D. W. Landow and J. A. Oliver, "Splanchnic Lactate Production in Cardiac Surgery Patients," Critical Care Medicine, Vol. 21, Supplement 2, 1993, pp. S84-S91.

[7] J. Mandak, et al., "Impact of Cardiopulmonary Bypass on Peripheral Tissue Metabolism and Microvascular Blood Flow," Perfusion, Vol. 23, No. 6, 2008, pp. 339-346. doi:10.1177/0267659109105359

[8] J. Mand'ak, et al., "Changes in Metabolism and Blood Flow in Peripheral Tissue (Skeletal Muscle) during Cardiac Surgery with Cardiopulmonary Bypass: The Biochemical Microdialysis Study," Perfusion, Vol. 19, No. 1, 2004, pp. 53-63. doi:10.1191/0267659104pf704oa 
[9] Y. A. Louagie, et al., "Continuous Cold Blood Cardioplegia Improves Myocardial Protection: A Prospective Randomized Study," The Annals of Thoracic Surgery, Vol. 77, No. 2, 2004, pp. 664-671. doi:10.1016/S0003-4975(03)01522-4

[10] J. Poling, et al., "Evaluation of Myocardial Metabolism with Microdialysis during Bypass Surgery with Cold Blood- or Calafiore Cardioplegia," European Journal Cardio-Thoracic Surgery, Vol. 30, No. 4, 2006, pp. 597603.

[11] E. Solligard, et al., "Rectal Lactate Levels in Endoluminal Microdialysate during Routine Coronary Surgery," Anaesthesia, Vol. 62, No. 3, 2007, pp. 250-258. doi:10.1111/j.1365-2044.2006.04937.x

[12] H. Lin and M. S. Suleiman, "Cariporide Enhances Lactate Clearance upon Reperfusion but Does Not Alter Lactate Accumulation during Global Ischaemia," Pflügers Archiv, Vol. 447, No. 1, 2003, pp. 8-13. doi:10.1007/s00424-003-1134-8

[13] J. Takala, E. Ruokonen and J. Takala, "Lactate Metabolism and Regional Lactate Exchange after Cardiac Surgery," New Horizons, Vol. 4, No. 4, 1996, pp. 483-492. doi:10.1007/BF01728327

[14] S. Inoue, M. Kuro and H. Furuya, "What Factors Are Associated with Hyperlactatemia after Cardiac Surgery Characterized by Well-Maintained Oxygen Delivery and a Normal Postoperative Course? A Retrospective Study," European Journal of Anaesthesiology, Vol. 18, No. 9, 2001, pp. 576-584.

[15] M. Ranucci, et al., "Hyperlactatemia during Cardiopulmonary Bypass: Determinants and Impact on Postoperative Outcome," Critical Care, Vol. 10, No. 6, 2006, p. R167. doi:10.1186/cc5113

[16] F. Toraman, et al., "Lactic Acidosis after Cardiac Surgery Is Associated with Adverse Outcome," Heart Surgery Forum, Vol. 7, No. 2, 2004, pp. E155-E159. doi:10.1532/HSF98.20041002

[17] A. Morishita, "Efficacy of Terminal Warm Blood Cardioplegia in Combination with Bretschneider-HTK Solution for Myocardial Protection," Kyobu Geka, Vol. 52, No. 10, 1999, pp. 831-835.

[18] J. Ackemann, W. Gross, M. Mory, M. Schaefer and M. M. Gebhard, "Celsior versus Custodiol: Early Postischemic Recovery after Cardioplegia and Ischemia at 5 Degrees C," The Annals of Thoracic Surgery, Vol. 74, No. 2, 2002, pp. 522-529. doi:10.1016/S0003-4975(02)03675-5
[19] N. Greenson, et al., "Usefulness of Cardiac Troponin I in Patients Undergoing Open Heart Surgery," American Heart Journal, Vol. 141, 2001, No. 3, pp. 447-455.

[20] M. Pojar, "Peripheral Tissue Metabolism during Off-Pump versus On-Pump Coronary Artery Bypass Graft Surgery: The Microdialysis Study," European Journal CardioThoracic Surgery, Vol. 33, No. 5, 2008, pp. 899-905. doi:10.1016/j.ejcts.2008.01.039

[21] M. Licker, A. Schweizer and F. E. Ralley, "Thermoregulatory and Metabolic Responses Following Cardiac Surgery," European Journal of Anaesthesiology, Vol. 13, No. 5, 1996, pp. 502-510 doi:10.1046/j.1365-2346.1996.00018.x

[22] A. Thoren, M. Elam and S. E. Ricksten, "Jejunal Mucosal Perfusion Is Well Maintained during Mild Hypothermic Cardiopulmonary Bypass in Humans," Anesthesia \& Analgesia, Vol. 92, No. 1, 2001, pp. 5-11. doi:10.1097/00000539-200101000-00003

[23] M. Heringlake, "The Metabolic and Renal Effects of Adrenaline and Milrinone in Patients with Myocardial Dysfunction after Coronary Artery Bypass Grafting," Critical Care, Vol. 11, No. 2, 2007, p. R51. doi:10.1186/cc5904

[24] M. A. Levin, et al., "Early On-Cardiopulmonary Bypass Hypotension and Other Factors Associated with Vasoplegic Syndrome," Circulation, Vol. 120, No. 17, 2009, pp. 1664-1671. doi:10.1161/CIRCULATIONAHA.108.814533

[25] D. W. Landry and J. A. Oliver, "The Pathogenesis of Vasodilatory Shock," The New England Journal of Medicine, Vol. 345, No. 8, 2001, pp. 588-589. doi:10.1056/NEJMra002709

[26] H. Janssen, P. H. E. Janssen and C. E. Broelsch, "UW Is Superior to Celsior and HTK in the Protection of Human Liver Endothelial Cells against Preservation Injury," Liver Transplantation, Vol. 10, No. 12, 2004, pp. 15141523. doi:10.1002/lt.20309

[27] G. Puhl, et al., "24-h Storage of Pig Livers in UW, HTK, Hydroxyethyl Starch, and Saline Solution: Is Microdialysis an Appropriate Method for the Continuous Graft Monitoring during Preservation?" Transplant International, Vol. 19, No. 4, 2006, pp. 303-309. doi:10.1111/j.1432-2277.2006.00289.x

[28] P. Panzera, "Solutions for Organ Perfusion and Storage: Haemorheologic Aspects," Transplantation Proceedings, Vol. 37, No. 6, 2005, pp. 2456-2458. 